\title{
Debriefing Experience Scale: Development of a Tool to Evaluate the Student Learning Experience in Debriefing
}

Shelly Jensen Reed

Brigham Young University - Provo, shelly-reed@byu.edu

Follow this and additional works at: https://scholarsarchive.byu.edu/facpub

Part of the Other Nursing Commons

\section{Original Publication Citation}

Reed, S. J. (2012). Debriefing Experience Scale: Development of a tool to evaluate the student learning experience in debriefing. Clinical Simulation in Nursing, 8(6), e211-e217.

\section{BYU ScholarsArchive Citation}

Reed, Shelly Jensen, "Debriefing Experience Scale: Development of a Tool to Evaluate the Student Learning Experience in Debriefing" (2012). Faculty Publications. 5321.

https://scholarsarchive.byu.edu/facpub/5321 


\title{
Debriefing Experience Scale: Development of a Tool to Evaluate the Student Learning Experience in Debriefing
}

\author{
Shelly J. Reed, DNP, APRN* \\ Brigham Young University, 530 SWKT, Provo, UT 84602, USA
}

\section{KEYWORDS \\ debriefing; \\ debriefing experience; \\ instrument; \\ learning; \\ nursing education; \\ psychometric testing; \\ simulation; \\ debriefing experience scale}

\begin{abstract}
Background: Debriefing represents the reflection phase of the simulation process, in which feelings are resolved and learning is solidified, but the nursing student experience during debriefing is largely unknown.

Method: The Debriefing Experience Scale was developed from debriefing literature and expert opinion. This scale was used in 2 research studies and refined through the use of factor analysis.

Results: The resulting scale, divided into 4 subscales, consists of 20 items defining the nursing student debriefing experience.

Conclusions: The Debriefing Experience Scale has the potential to assist in providing further insight.

\section{Cite this article:}

Reed, S. J. (2012, July/August). Debriefing experience scale: Development of a tool to evaluate the student learning experience in debriefing. Clinical Simulation in Nursing, 8(6), e211-e217. doi:10.1016/j.ecns.2011.11.002.

(C) 2012 International Nursing Association for Clinical Simulation and Learning. Published by Elsevier Inc. All rights reserved.
\end{abstract}

\section{Introduction}

The use of human patient simulation in nursing education has significantly increased over the past 10 years (Bremner, Aduddell, Bennett, \&VanGeest, 2006; Howard, Englert, Kameg, \& Perozzi, 2011; Kaakinen \& Arwood, 2009; Nehring \& Lashley, 2004; Starkweather \& KardongEdgren, 2008). Simulation as a teaching strategy is a means for students to practice clinical skills or for educators to assess and evaluate student skills (Cantrell, 2008). A simulated learning environment is an ideal setting for students to learn decision-making and psychomotor skills without risk of inflicting harm to patients, providing a safe and effective

\footnotetext{
* Corresponding author: shelly-reed@byu.edu (S. J. Reed).
}

means of preparing nurses for practice (Broussard, 2008; Jeffries, 2006).

Feedback, including debriefing, has been identified as the most important part of simulation-based education in medical education (Fanning \& Gaba, 2007). In nursing education, debriefing has been viewed as central to simulation learning strategies (Neill \& Wotton, 2011). This article describes the development and initial testing of an instrument to evaluate the nursing student debriefing experience.

\section{Background}

Theoretical frameworks have been applied to simulation in nursing education. Jeffries (2003) describes use of a framework to guide the designing, implementing and evaluating 
of simulations in nursing. Other theories are used to describe the learning provided by simulation. One, Kolb's experiential learning theory, describes cyclical learning, consisting of a concrete experience, reflective observation, abstract conceptualization, and active experimentation (Fanning \& Gaba, 2007). Simulation learning
Key Points

- Debriefing is the reflective period following enactment of a simulation scenario.

- Simulation experts agree learning is solidified during debriefing. the nursing student experience during debriefing; this article provides seminal information to begin to fill this knowledge gap.
- Little is known about is portrayed by this cycle as starting with an experience, a performance of a simulation scenario, followed by a reflective period, also known as debriefing.

Facilitated reflection as a part of debriefing provides meaning for the simulation experience. During debriefing, the abstract conceptualization phase in Kolb's framework begins. The team works together to think critically about the event and come up with a plan for similar future experiences. Ac- tive experimentation represents the final phase in the programmed learning provided by Kolb's framework. It is applied as the simulation participants take the learning provided by simulation and debriefing and transfer it to real world situations (Arafeh, Hansen, \& Nichols, 2010; Fanning \& Gaba, 2007; Kayes, Kayes, \& Kolb, 2005; Neill \& Wotton, 2011; Waldner \& Olson, 2007).

Debriefing literature also describes other aspects and benefits occurring through reflection, some of which align directly with Kolb's experiential learning theory. Debriefing has been portrayed as the most important part of a simulation. Time is allowed to "cool down" after the simulation performance. Debriefing aids simulation participants in understanding, analyzing, and synthesizing their thoughts, feelings, and actions during a simulation (Decker, 2007; Driefuerst, 2009; Fanning \& Gaba, 2007; Rudolph, Simon, Raemer, \& Eppich, 2008). Errors made during the simulation are identified, and a plan for improvement is developed (Salas et al., 2008). Debriefing also provides a venue where negative emotions can be addressed (Hill \& Lance, 2002). Baker, Jensen, and Kolb (1997) stress that debriefing as part of the educational process is "pivotal in transforming experience into learning."

While expert opinion abounds concerning debriefing in general, more research is needed concerning the simulation experience of students in undergraduate nursing education (Jeffries, 2006; Rhodes \& Curran, 2005). Research is particularly lacking in the area of debriefing, as minimal information is available in the nursing literature specific to the debriefing experience of nursing students (Neill \& Wotton, 2011). Examination of debriefing is needed in order to provide understanding of the learning process provided therein (Cantrell, 2008).
Instruments are often used during research to discriminate between experiences and are designed to measure characteristics on a continuum. When reliable and valid instruments are not available to measure a particular construct of interest, factor analysis can be used to help produce reliable and valid measures. One of the first steps in this process is to prepare an instrument that can be evaluated by factor analysis (Pett, Lackey, \& Sullivan, 2003). Factor analysis is a statistical method commonly used during instrument development to define relationships among large numbers of variables. Factors are related items that define part of a construct and are grouped together (DeVon et al., 2007).

\section{Scale Development}

\section{Content Validity}

Scale items are developed through a literature search, seeking expert opinions, and population sampling, as the researcher defines the construct of interest and its dimensions (DeVon et al., 2007). To develop the debriefing instrument, a comprehensive review of simulation literature was completed and components considered to be important to the debriefing experience were identified. From this literature search, the construct that learning takes place during the debriefing experience was derived. The components were formulated into items, and a tool was developed, the Debriefing Experience Scale, consisting of 37 items.

The newly formulated Debriefing Experience Scale was then sent to three nationally known nursing simulation experts, who reviewed it for content and wording. Peer evaluation of the original draft of a newly formed instrument provides a critique of the items, instructions, and appearance of an instrument (Pett et al., 2003). Through the review, wording changes were suggested to clarify items, and these suggestions were incorporated into the scale. Two additional items were identified by the experts and were added to the scale, for a total of 39 items. This helped to provide a large pool of initial items, important during the item analysis and subsequent correlations of items (DeVon et al., 2007; Pett et al., 2003). Each item was categorized in a pattern similar to existing National League of Nursing simulation scales, into the areas of (student) experience and importance (to the student), in essence creating two scales within one.

\section{Scoring and Subscales}

The two scales within the Debriefing Experience Scale are both rated with Likert-type rating, but with different labels for the anchors in each scale. The experience scale has Likert-type rating from 1 (strongly disagree) to 5 (strongly agree). The importance scale, while sharing the same 39 items as the experience scale, has Likert-type rating from 1 (not important) to 5 (very important). The 39 items in the initial scale were grouped into like items by the researcher, creating seven subscales. 
During instrument development, pilot testing should be undertaken by respondents selected from the same population of study (Pett et al., 2003). Pilot testing was performed when the initial version of the Debriefing Experience Scale was administered to undergraduate nursing students in a baccalaureate nursing program. Students used the Debriefing Experience Scale to rate their debriefing experience in the scale areas of experience and importance to the student. The debriefing types compared were oral discussion, journaling, and blogging.

Following Institutional Review Board approval, 100 undergraduate nursing students participated in the pilot study by returning informed consent and a completed copy of the scale. For additional content validity, a section for comments was included on the scale for input from students concerning scale clarity, item wording, and scale format. From the data collected, exploratory factor analysis and item analysis were performed. Factor analysis refers to statistical processes used to reduce a large amount of statistical data to a meaningful size. This process is a method that helps establish the correlation of the items. As a result of the analyses, 10 items of 39 were excluded from the scale because of high correlations between items, factor loadings $>0.80$ in more than one factor area, and factor loadings $<0.40$.

After factor analysis, 29 items were left in the overall scale. From suggestions found in student comments, spacing changes were made, wording was clarified, and all items were worded positively to assist students with scoring. For example, an item worded "I am uncomfortable expressing myself" was reworded positively to "I feel comfortable expressing myself." This was related to student comments about confusion on how to rate negatively worded items in the importance scale. The overall scale was again reviewed by one of the nationally known nursing simulation experts, focusing on spacing and clarity issues. Suggestions from this review were implemented. Previously labeled subscales from the first version of the scale were eliminated entirely. Items that were grouped during the exploratory factor analysis process were dispersed throughout the second version of the scale by the use of a table of random numbers. The items were listed 1 through 29 and were again categorized in the dual scale areas of student experience and importance of that experience to the student.

\section{Aim}

The aim of the study was to refine an instrument describing the nursing student experience during debriefing.

\section{Method}

\section{Sample}

The participants were nursing students $(N=130)$ in an undergraduate baccalaureate nursing program who were participating in obstetric $(N=75)$ and intensive care
$(N=55)$ simulations associated with their courses. Five men and 125 women participated. The students ranged in age from 19 to 51 years, with an average age of 22.2 years. As part of their nursing education prior to the study, all had participated in at least five simulations with debriefing sessions. None of the students had participated in the pilot testing of the instrument.

\section{Procedure}

Internal Institutional Review Board approval was obtained prior to the study. Both the obstetric and the intensive care courses had simulation exercises already incorporated into their curriculum. Following completion of the simulation scenario, student groups (from 5 to 8 students per group) were randomized as a group into one of two debriefing types: video-assisted oral discussion debriefing and oral discussion without video. Informed consent was not obtained prior to randomization, as debriefing was required to complete the simulation experience, and no clear evidence is available defining one debriefing type as more advantageous than another. Debriefing sessions were facilitated by the same course faculty who had led the simulation scenario and not by any member of the research team.

Following completion of debriefing, session participants were invited by a member of the research team to participate in the study and were assured that study participation was voluntary and independent of course requirements. Informed consent was given, and students who chose to participate in the study returned a completed informed consent and a completed Debriefing Experience Scale to a locked box in the simulation center. Each completed scale was separated from the informed consent and coded with an individual number to maintain student anonymity.

\section{Data Analysis}

Data were entered into PASW Statistics 18. Exploratory factor analysis was performed on the experience portion of the dual scale, followed by an oblimin rotation with Kaiser normalization. Exploratory factor analysis is used when the researcher does not know how many factors are necessary to explain interrelationships among a set of items (Pett et al., 2003). It is also suggested that exploratory factor analysis helps to develop and evaluate scales with a relatively untested population (Snelgrove \& Slater, 2003). Oblimin rotation was used in the study to identify item groupings. The oblique rotation produced by oblimin rotation allows for a degree of correlation among factors and produces a pattern matrix in the output (Snelgrove \& Slater, 2003). Factors were defined with items loading 0.4 or higher, with the meaning of each factor determined by the item loading most highly on it. A sample size large enough to reduce standard error of the correlations is 
identified as a minimum of between 100 and 200 participants, with a participant-to-variable ratio of at least 2:1 (Snelgrove \& Slater, 2003). There were 29 variables (items) in this study and 130 participants, satisfying these requirements.

\section{Results}

\section{Factor Analysis}

During the factor analysis process, nine items that either loaded at less than 0.4 or correlated too highly with other items were eliminated. With the remaining 20 items, an oblimin rotation converged after 35 iterations, and four factors emerged (Table 1). These 20 items were included in the final version of the scale and are rated in both the dual scale areas of experience and importance. Items in the four factor areas were then identified as subscales on the final version of the scale. A Likert-type rating, from 1 to 5 , is present for all 20 items in both scale areas.

\section{Internal Consistency}

Cronbach's alpha coefficient is the preferred approach to the estimate of internal consistency reliability (DeVon et al., 2007; Pett et al., 2003). In an instrument and subscales, values of .80 or higher are seen as highly desirable (Larson, Franzén-Dahlin, Billing, Murray, \& Wredling, 2005). Cronbach's alpha was determined for each part (experience and importance) of the dual scale, with the Cronbach's alpha for all items in the scale area of experience as .93, and the Cronbach's alpha for all items in the scale area of importance at .91. Alphas for the two larger scales within the Debriefing Experience Scale (experience and importance), and for the subscales, are found in Table 2.

\section{Discussion}

The two scales in the Debriefing Experience Scale were developed to measure (a) the student experience during debriefing and (b) the importance of those experiences to the student. Although another instrument concerning debriefing does exist, the focus of the student version allows students to rate their instructors on the quality of a debriefing (Center for Medical Simulation, 2010) rather than the student participant experience during the debriefing itself. As debriefing is emphasized as important to maximize learning and as a part of experiential learning (Dieckmann, Molin Friis, Lippert, \& Ostergaard, 2009; Fanning \& Gaba, 2007; Kayes et al., 2005), understanding the learning experience of the actual debriefing participant is essential.

\section{Scale Items}

The nursing student debriefing experience is identified by the remaining 20 scale items in the Debriefing Experience
Scale (Table 3). While most items are substantiated as part of the debriefing experience by experts in the literature, the elimination of some items through the factor analysis process was surprising.

The subscale Analyzing Thoughts and Feelings contains four items related to emotional, psychological, behavioral, and environmental aspects of debriefing. These components are generally mirrored in the description of guided reflection by Jeffries (2006), in which debriefing is identified as a time for learners to reflect on their experience, emotions, and clinical competency. Debriefing is defined as a place to address students' emotions and affirm feelings (Cantrell, 2008), also addressed by the items in this subscale.

There are eight items in the second subscale, Learning and Making Connections. These items highlight areas of debriefing that promote learning in the experience of the debriefing participant. The focus on learning during debriefing found in this subscale is also emphasized in many articles on debriefing. For example, debriefing is described as a venue for learning and as a crucial step in experiential learning in which insights are developed and integrated into future actions (Nehring, Ellis, \& Lashley, 2001; Rudolph, Simon, Rivard, Dufresne, \& Raemer, 2007; Rudolph et al., 2008). There is a general consensus in all debriefing literature that learning takes place during the debriefing and reflection processes, and items that factored into this category also substantiate these claims.

Of note are items concerning learning that seem very similar but represent a different variable to students as determined by the factor analysis process. For example, the items "Debriefing helped me to make connections in my learning" and "Debriefing helped me to make connections between theory and real-life situations" both discuss the connecting of ideas that occurs during the learning process. However, the first item discusses the process itself, while the second represents the connection of ideas that occurs between theoretical learning and real-life situations represented by the simulation scenario.

The subscale Facilitator Skill in Conducting the Debriefing contains five items specifically related to perceived facilitator skill in managing the debriefing. Time management and facilitator skill are themes in this subscale area. Facilitator skill in debriefing is the emphasis of a wellknown tool concerning debriefing (Center for Medical Simulation, 2010). The importance of facilitator skill is supported in the Debriefing Experience Scale by the fact that after factor analysis, almost half of the items left to define the student debriefing experience are related to the debriefing session facilitator. Items in this subscale are directly related to the facilitator's skill in managing the time and structure of the debriefing session, as well as the importance to the student experience of having a facilitator who is a content expert.

The final identified subscale is directed toward the facilitator's guidance during the session. This subscale, 
Table 1 Factor Loadings of Items*

\begin{tabular}{|c|c|c|c|c|}
\hline Items & Factor 1 & Factor 2 & Factor 3 & Factor 4 \\
\hline The facilitator taught... & .68 & & & \\
\hline The facilitator provided constructive... & .58 & & & .431 \\
\hline Debriefing helped me to make connections... & & .88 & & \\
\hline Debriefing was helpful in processing... & & .78 & & \\
\hline Debriefing provided me with a learning... & & .76 & & \\
\hline I became more aware of myself... & & .60 & & \\
\hline Debriefing helped me to clarify... & & .50 & & \\
\hline Debriefing helped me to make connections... & & .46 & & \\
\hline Debriefing helped me to analyze my thoughts & & & .69 & \\
\hline The facilitator reinforced aspects of behavior... & & & .64 & \\
\hline Debriefing allowed me a means to reflect... & & & & .49 \\
\hline I had enough time to debrief... & & & & .45 \\
\hline The session facilitator was an expert... & & & & .43 \\
\hline
\end{tabular}

Table 2 Reliability Scores for Subscales

\begin{tabular}{lllc}
\hline Subscale & $\begin{array}{l}\text { Cronbach's Alpha: } \\
\text { Experience Items }\end{array}$ & $\begin{array}{c}\text { Cronbach's Alpha: } \\
\text { Importance Items }\end{array}$ & $\begin{array}{c}\text { Number of Items } \\
\text { in Scale/Subscale }\end{array}$ \\
\hline Overall scale & .93 & .91 & 20 \\
Analyzing thoughts and feelings & .80 & .61 & .85 \\
Learning and making connections & .89 & .75 & 8 \\
Facilitator skill in conducting the debriefing & .80 & .65 & 3 \\
Appropriate facilitator guidance & .84 & & 3 \\
\hline
\end{tabular}

Appropriate Facilitator Guidance, consists of three items. The focus is still on the facilitator in this subscale, emphasizes the finesse of the facilitator in guiding the debriefing. Of note is the differentiation of two seemingly similar items in the last two subscales: "The debriefing session facilitator talked the right amount during debriefing" and "The facilitator taught the right amount during debriefing." The process of factor analysis eliminates items that are highly correlated with each other, in essence eliminating items that are just a restatement of another item. The fact that these two seemingly similar items survived a two-step factor analysis process shows that students perceive facilitator talking and teaching differently in their debriefing experience.

Several items were eliminated from the original scale through the two-step factor analysis process (Table 4). These items were eliminated for two reasons. First, the item may have been highly correlated with another item, meaning the two items expressed basically the same concept. For example, the correlation between the two items
"The facilitator reinforced aspects of my behavior" and "The facilitator reinforced aspects of the health care team's behavior" was high (0.8), meaning they are essentially the same item. Between the two, the item "The facilitator reinforced aspects of my behavior" loaded lower on structure rotation and thus was eliminated from the scale.

Second, the item may have loaded too low (less than 0.4) on structure rotation altogether, meaning the item was not strong enough to support its being an identified part of the student debriefing experience. Two examples of factors that did not load above the 0.4 mark are "Importance of trust was emphasized" and "I felt comfortable expressing myself." One item, "Watching myself on video helped me see where I needed to improve," was either marked not applicable or not answered on more than half the completed surveys and is not included in the final version of the scale.

The items on the Debriefing Experience Scale that have been identified provide a window into the debriefing experience for students and aspects of learning that occur during debriefing. Additional research is needed to 


\section{Table 3 Debriefing Experience Scale Subscales and Items}

Analyzing thoughts and feelings

1. Debriefing helped me to analyze my thoughts.

2. The facilitator reinforced aspects of the health care team's behavior.

3. The debriefing environment was physically comfortable.

4. Unsettled feelings from the simulation were resolved by debriefing.

Learning and making connections

1. Debriefing helped me to make connections in my learning.

2. Debriefing was helpful in processing the simulation experience.

3. Debriefing provided me with a learning opportunity.

4. Debriefing helped me to find meaning in the simulation.

5. My questions from the simulation were answered by debriefing.

6 . I became more aware of myself during the debriefing session.

7. Debriefing helped me to clarify problems.

8. Debriefing helped me to make connections between theory and real-life situations.

Facilitator skill in conducting the debriefing

1. The facilitator allowed me enough time to verbalize my feelings before commenting.

2. The debriefing session facilitator talked the right amount during debriefing.

3. Debriefing provided a means for me to reflect on my actions during the simulation.

4. I had enough time to debrief thoroughly.

5. The debriefing session facilitator was an expert in the content area.

Appropriate facilitator guidance

1. The facilitator taught the right amount during the debriefing session.

2. The facilitator provided constructive evaluation of the simulation during debriefing.

3. The facilitator provided adequate guidance during the debriefing.

investigate and define the debriefing experience further, especially with the burgeoning use of simulation in health care education. Research is needed to identify which debriefing methods and techniques contribute most to student learning. As experts agree that debriefing is key to the learning provided by the simulation, it is vital to facilitate the best possible experience during debriefing in order for students to maximize their learning.

Additional psychometric testing is also needed on the Debriefing Experience Scale to ensure it reflects the student nurse debriefing experience and the importance of those experiences to the student. Confirmatory factor analysis follows exploratory factor analysis, including theoretical knowledge to further test the construct validity of the tool and validate the extent to which the statistical model fits the data. Test-retest reliability, administering the same test to the same group of respondents at different times, could also be used, in addition to Cronbach's alpha coefficient
Table 4 Items Eliminated from the Debriefing Experience Scale during Factor Analysis

1. I felt safe during debriefing.

2. Importance of trust was emphasized.

3. I felt comfortable expressing myself.

4. The debriefing environment was emotionally comfortable.

5. Learning objectives were made clear to me at the beginning of the debriefing session.

6. I learned more during debriefing than in the simulation session.

7. Watching myself on video helped me see where I needed to improve.

8. During debriefing, I was able to make connections between myself and others.

9. Debriefing provided a bridge for the gaps between my (or my group's) performance.

10. Emotions that came up during the simulation were addressed.

11. Debriefing provided a "cooling down" period after the simulation.

12. I was clear about my role as a nurse following debriefing.

13. Debriefing helped me to understand my professional nursing values.

14. Debriefing allowed me a chance to "de-role" or step back from my simulated role as a nurse to that of being a student.

15. Debriefing helped me to understand the perspective of others.

16. I was clear about my role as team member following debriefing.

17. Debriefing helped me to analyze my performance.

18. The facilitator reinforced aspects of my behavior.

19. The facilitator used open-ended questions.

calculations to establish reliability of the scale (DeVon et al., 2007). Testing the scale with debriefing participants other than nursing students could provide reliability and validity for those populations and expand venues for use of the scale.

Factor analysis and testing (other than Cronbach's coefficient alpha) were done only on the experience portion of the dual scale. Psychometric testing needs to be done to determine reliability and validity of the importance portion. Another option for the researcher would be to use only the experience portion of the scale if the importance portion is not needed to answer any research questions.

The Debriefing Experience Scale holds the potential to investigate the nursing student debriefing experience in multiple ways. Possibilities include comparing the student debriefing experience between different debriefing types or a combination of debriefing types, evaluating the student debriefing experience as conducted by session facilitators with different training, comparing the experience in debriefing sessions of different length, and so forth. The scale could also be used with other participant types, such as experienced nurses, or with other health care professionals; however, reliability would have to be reevaluated in any population 
outside of nursing students. With the potential to increase learning during this key component of the overall simulation process, further investigation seems well worth the effort.

\section{References}

Arafeh, J. M. R., Hansen, S. S., \& Nichols, A. (2010). Debriefing in simulated-based learning: Facilitating a reflective discussion. Journal of Perinatal \& Neonatal Nursing, 24(4), 302-309.

Baker, A. C., Jensen, P. J., \& Kolb, D. A. (1997). In conversation: Transforming experience into learning. Simulation \& Gaming, 28(1), 6-12.

Bremner, M. N., Aduddell, K., Bennett, D. N., \& VanGeest, J. B. (2006). The use of human patient simulators. Nurse Educator, 31(4), 170-174.

Broussard, L. (2008). Simulation-based learning: How simulations help nurses improve clinical skills and preserve patient safety. Nursing for Women's Health, 12(6), 521-524.

Cantrell, M. A. (2008). The importance of debriefing in clinical simulations. Clinical Simulation in Nursing, 4, 19-23. doi: 10.1016/j.ecns.2008.06.006.

Center for Medical Simulation. (2010). Debriefing Assessment for Simulation in Healthcare (DASH). Retrieved November 10, 2010, from http://www. harvardmedsim.org/debriefing-assesment-simulation-healthcare.php

Decker, S. (2007). Integrating guided reflection into simulated learning experiences. In P. R. Jeffries (Ed.), Simulation in nursing education: From conceptualization to evaluation. New York, NY: National League for Nursing.

DeVon, H. A., Block, M. E., Moyle-Wright, P., Ernst, D. M., Hayden, S. J., Lazzara, D. J., et al. (2007). A psychometric toolbox for testing validity and reliability. Journal of Nursing Scholarship, 39(2), 155-164.

Dieckmann, P., Molin Friis, S., Lippert, A., \& Ostergaard, D. (2009). The art and science of debriefing in simulation: Ideal and practice. Medical Teacher, 31, 287-294.

Driefuerst, K. T. (2009). The essentials of debriefing in simulation learning: A concept analysis. Nursing Education Perspectives, 30(2), 109-114.

Fanning, R. M., \& Gaba, D. M. (2007). The role of debriefing in simulation-based learning. Simulation in Healthcare, 2(1), 1-9.

Hill, J. L., \& Lance, C. G. (2002). Debriefing stress. Simulation \& Gaming, 33(4), 490-503.

Howard, V. M., Englert, N., Kameg, K., \& Perozzi, K. (2011). Integration of simulation across the undergraduate curriculum: Student and faculty perspectives. Clinical Simulation in Nursing, 7(1), e1-e10. doi: 10.1016/j.ecns.2009.10.004.

Jeffries, P. R. (2003). Designing, implementing, and evaluating simulations used as teaching strategies in nursing. Nursing Education Perspectives, 26(2), 96-103.
Jeffries, P. R. (2006). Designing simulations for nursing education. Annиal Review of Nursing Education, 4, 106-114.

Kaakinen, J., \& Arwood, E. (2009). Systematic review of nursing simulation literature for use of learning theory. International Journal of Nursing Education Scholarship, 6(1), 1-20.

Kayes, A. B., Kayes, D. C., \& Kolb, D. A. (2005). Experiential learning in teams. Simulation \& Gaming, 36(3), 330-354.

Larson, J., Franzén-Dahlin, A., Billing, E., Murray, V., \& Wredling, R. (2005). Spouse's life situation after partner's stroke event: Psychometric testing of a questionnaire. Journal of Advanced Nursing, 52(3), 300-306.

Nehring, W. M., Ellis, W. E., \& Lashley, F. R. (2001). Human patient simulators in nursing education: An overview. Simulation \& Gaming, 32(2), 194-204.

Nehring, W. M., \& Lashley, F. R. (2004). Human patient simulators in nursing education: An international survey. Nursing Education Perspectives, 25(5), 244-248.

Neill, M. A., \& Wotton, K. (2011). High-fidelity simulation debriefing in nursing education: A literature review. Clinical Simulation in Nursing, 7, e161-e168. doi:10.1016/j.ecns.2011.02.001.

Pett, M. A., Lackey, N. R., \& Sullivan, J. J. (2003). Making sense of factor analysis: The use of factor analysis for instrument development in health care research. Thousand Oaks, CA: Sage.

Rhodes, M. L., \& Curran, C. (2005). Use of the human patient simulator to teach clinical judgment skills in a baccalaureate nursing program. CIN: Computers, Informatics, Nursing, 23(5), 256-262.

Rudolph, J. W., Simon, R., Raemer, D. B., \& Eppich, W. J. (2008). Debriefing as formative assessment: Closing performance gaps in medical education. Academy of Emergency Medicine, 15(11), 1010-1016.

Rudolph, J. W., Simon, R., Rivard, P., Dufresne, R. L., \& Raemer, D. B. (2007). Debriefing with good judgment: Combining rigorous feedback with genuine inquiry. Anesthesiology Clinics, 25, 361-376.

Salas, E., Klein, C., King, H., Salisbury, M., Augenstein, J. S., Birnbach, D. J., et al. (2008). Debriefing medical teams: 12 evidencebased best practices and tips. Joint Commission Journal on Quality and Patient Safety, 34(9), 518-527.

Snelgrove, S., \& Slater, J. (2003). Approaches to learning: Psychometric testing of a study process questionnaire. Methodological Issues in Nursing Research, 43(5), 496-505.

Starkweather, A. R., \& Kardong-Edgren, S. (2008). Diffusion of innovation: Embedding simulation into nursing curricula. International Journal of Nursing Education Scholarship, 5(1), 1-11.

Waldner, M. H., \& Olson, J. K. (2007). Taking the patient to the classroom: Applying theoretical frameworks to simulation in nursing education. International Journal of Nursing Education Scholarship, 4(1), 1-14. 